\title{
Unstable angina due to spontaneous left internal mammary artery dissection seventeen years after coronary artery bypass graft surgery: A case report and literature review
}

\author{
Vincenzo I nfantino, Claudio Moretti, Pierluigi Omedè \\ Division of Cardiology, Department of Internal Medicine, Città Della Salute e Della Scienza, Turin, I taly
}

Correspondence: Vincenzo Infantino. Address: University of Turin, Città della Salute e della Scienza, C.so Bramante 88, Turin, Italy. Email: vincenzo.infantino@alice.it

Received: February 17, 2014

Accepted: April 28, 2014

Online Published: May 5, 2014

DOI : $10.5430 /$ crim.v1n2p102

URL: http://dx.doi.org/10.5430/crim.v1n2p102

\section{Abstract}

A 64 year-old male was admitted to the emergency department for multiple episodes of chest pain at rest. His cardiac history began seventeen years before when a coronary artery bypass grafting (CABG) was performed. This included left internal mammary artery (LIMA) graft to left anterior descending artery (LAD) and saphenous vein graft (SVG) to the diagonal branch. Since then the patient was subjected to numerous percutaneous coronary angioplasties on LAD, circumflex artery and SVG. The last angiographic control showed LIMA graft patency.

The coronary angiography showed LAD, circumflex and right coronary artery occlusion and LIMA graft critical stenosis with evidence of dissection. Then, the lesion was crossed and intravascular ultrasound (IVUS) evaluation was performed to investigate the extent of the dissection. IVUS demonstrated dissection with an intramural hematoma and presence of intimal tear. Therefore, a coronary angioplasty with multiple direct drug eluting stents implantation completed with post-dilatation was performed. Good angiographic and IVUS results were achieved.

Spontaneous LIMA graft dissection is a rare condition. We reported the ninth case in literature and the one with the highest latency period between CABG and LIMA graft dissection. This situation may lead to an acute coronary syndrome, an unstable angina in our report, therefore prompt treatment, percutaneous or surgical, is decisive.

Spontaneous arterial graft dissection is sometimes seen in women, especially in the post-partum period, it usually occurs during the fifth and sixth decade in patients affected by arterial hypertension, vasculitis, connective tissue disorders or atherosclerotic disease.

\section{Keywords}

Unstable angina, Spontaneous left internal mammary artery dissection

\section{I ntroduction}

Left internal mammary artery (LIMA) coronary artery bypass grafting is a consolidated procedure in patients undergoing revascularization for coronary artery disease as the graft is rarely involved by atherosclerotic disease. Moreover LIMA grafts are durable and have a low rate of related complications ${ }^{[1]}$. 
Spontaneous LIMA dissection is an extremely rare event and commonly occurs as a complication of interventional manipulation mainly in the early post-operative time, although some cases have been described at a distance of few years after coronary artery bypass grafting ${ }^{[2]}$.

Here we describe a case of spontaneous LIMA dissection occurred seventeen years after coronary artery bypass grafting, assessed by angiographic and IVUS methods and treated with percutaneous angioplasty and stents implantation. Such a long period of time for the progression of spontaneous LIMA dissection has not previously been reported in literature.

\section{Case presentation}

A 64 year-old male, former smoker, suffering from arterial hypertension, diabetes mellitus in treatment with oral hypoglycemic agents and dyslipidemia, was admitted to the emergency department for multiple episodes of chest pain at rest. His cardiac history began twenty-four years before with an inferior acute myocardial infarction treated on medical therapy. Because of recurrent angina and multivessel coronary artery disease detection by coronary angiography, after seven years a coronary artery bypass grafting was performed. This included LIMA graft to his left anterior descending artery (LAD) and saphenous vein graft (SVG) to the diagonal branch.

Since then for appearance of exertional angina the patient was subjected to numerous percutaneous coronary angioplasties with multiple drug eluting stents implantation on LAD, circumflex artery and SVG. The last angiographic control in 2010 showed LIMA graft patency (see Figure 1, Panel A).

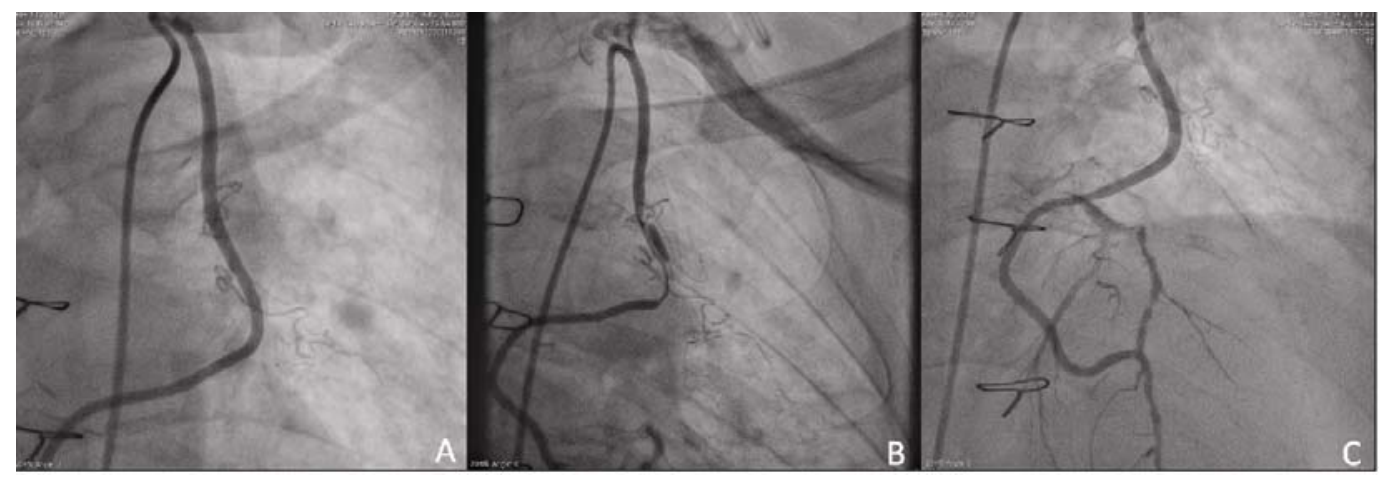

Figure 1. Panel A: LIMA graft patency (2010); Panel B: spontaneous body LIMA graft dissection with parietal hematoma; Panel C: good angiographic results after stents implantation. LIMA: left internal mammary artery.

In July 2013 because of the reappearance of at rest and on exertion angina the patient was hospitalized. The electrocardiogram showed no further ischemic alterations compared to the previous ones, furthermore there was no increase in troponin levels.

The coronary angiography showed total occlusion of middle segment of LAD, of middle segment of circumflex artery and of proximal tract of right coronary artery. Moreover, the exam revealed diffuse SVG atherosclerotic disease with subcritical body stenosis and distal critical one. At the level of the body of LIMA graft there was a critical stenosis with evidence of spontaneous dissection and parietal hematoma (see Figure 1, Panel B). Then, the lesion was crossed with a guide wire, and intravascular ultrasound (IVUS) evaluation was performed to investigate the extent of the dissection and to confirm the correct placement of the guide wire. IVUS demonstrated dissection with an intramural hematoma and presence of intimal tear (see Figure 2, Panel A and B). Therefore a coronary angioplasty with multiple direct drug eluting stents implantation completed with post-dilatation was performed. The angiographic and IVUS controls (see Figure 2, 
Panel C) confirmed good results with complete resolution of dissection and correct stents apposition (see Figure1, Panel C). Few days after the procedure, the patient was discharged asymptomatic.

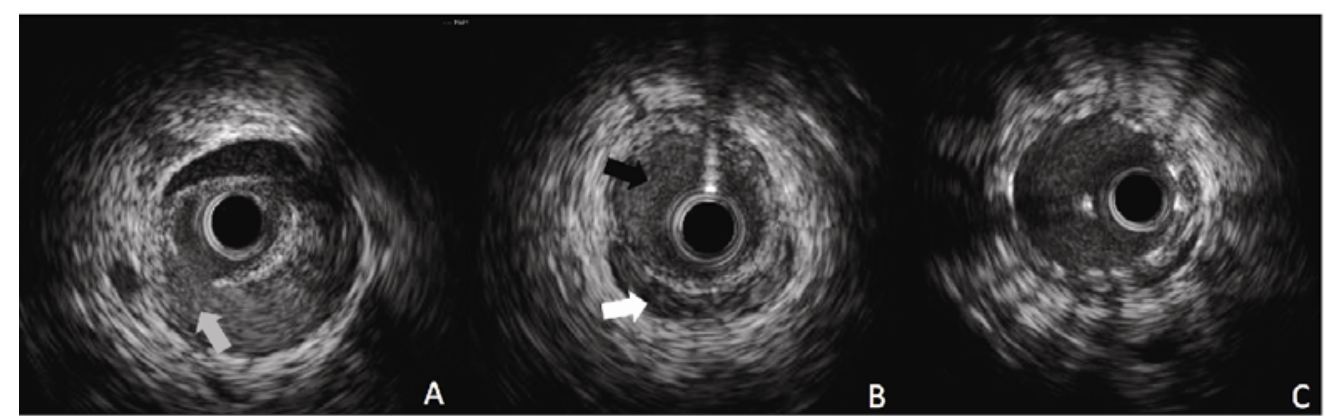

Figure 2. Panel A and B: IVUS evaluation of LIMA graft showed dissection with intimal tear (grey arrow), true (black arrow) and false lumen (white arrow); Panel C: IVUS control after stents implantation. IVUS: intravascular ultrasound; LIMA: left internal mammary artery.

\section{Discussion}

Patency rates of LIMA grafts to left anterior descending (LAD) are very positive over a ten year period of time, though rarely graft failure may occur ${ }^{[1]}$. The LIMA dissection generally occurs as a complication of interventional manipulation although graft extensive kinking during surgical intervention, probably as a result of the deforming effect of jet stream and flow turbulence, may also cause dissection. This may occur while harvesting the graft from the chest wall and making the anastomotic site ${ }^{[1]}$. Spontaneous LIMA dissection is a rare condition, in fact our case is the ninth described in literature and it is the one with the highest latency period between coronary artery bypass grafting and LIMA dissection.

Risk factors for spontaneous arterial graft dissection are the same proposed for spontaneous coronary dissection. It is usually seen in women, especially in the post-partum period ${ }^{[4-6]}$. It usually occurs during the fifth and sixth decade, while other risk factors are represented by arterial hypertension, vasculitis, connective tissue disorders and atherosclerotic disease ${ }^{[2]}$. However, spontaneous dissection has been reported in patients after vigorous exercise and lifting a heavy object ${ }^{[3]}$.Therefore, although the LIMA dissection represents an infrequent adverse event in clinical practice, it is essential to consider it when there is resumption of angina or when acute coronary syndrome occurs, in patients who have previously undergone surgical revascularization and who have important comorbidities or risk factors that may predispose to spontaneous arterial graft dissection.

Patency rates following percutaneous intervention on the LIMA graft have been favorable. Ziaee et al. reported a 7.4\% restenosis rate in patients undergoing balloon angioplasty with or without stenting versus a $15 \%-26 \%$ restenosis rate when a surgical intervention was performed ${ }^{[1]}$.

\section{Conclusion}

Spontaneous LIMA graft dissection is rarely seen but it can lead to an acute coronary syndrome, an unstable angina in our report, therefore prompt treatment, percutaneous or surgical, is decisive ${ }^{[2,7,8]}$.

\section{References}

[1] Ziaee A, Puri S, Kern MJ. Stenting for spontaneous left internal mammary artery dissection: a case report. Catheter Cardiovasc Interv. 2003 Nov; 60(3): 389-91. http://dx.doi.org/10.1002/ccd.10666 
[2] Karabulut A, Tanriverdi S. Acute coronary syndrome secondary to spontaneous dissection of left internal mammary artery by-pass graft nine years after surgery. Kardiol Pol. 2011; 69(9): 970-2; discussion 973.

[3] Suresh V, Evans S. Successful stenting of stenotic lesion and spontaneous dissection of left internal mammary artery graft. BMJ Case Rep. 2009 Feb; 16. http://dx.doi.org/10.1136/bcr.2006.087551

[4] Koutouzis M, Mavrogeni S, Nikolidakis S, Lazaris E, Kyriakides ZS. Multislice computed tomographic angiography of a spontaneous left internal mammary graft dissection. Int J Cardiol. 2007 Jan 18; 114(3): 384-5. Epub 2006 Apr 19. http://dx.doi.org/10.1016/j.ijcard.2005.11.075

[5] Wong P, Rubenstein M, Inglessis I, Pomerantsev E, Ferrell M, Leinbach R. Spontaneous spiral dissection of a LIMA-LAD bypass graft: a case report. J Interv Cardiol. 2004 Aug; 17(4): 211-3. http://dx.doi.org/10.1111/j.1540-8183.2004.02085.x

[6] Claessens P, Willaert W, Claessens M, Bloemen H, Coosemans M, van Eeuwijk J, et al. Symptomatic atraumatic dissection of a left internal mammary artery graft five years after coronary artery bypass graft surgery. Can J Cardiol. 2002 Jul; $18(7)$ : 759-61.

[7] Qaddour A, Roongsritong C. Spontaneous dissection bilateral internal mammary arterial grafts. J Invasive Cardiol. 2002 Apr; 14(4): 207-8.

[8] Claessens P, Janssens L, Vanderheyden M. Unstable angina due to spontaneous dissection of a LIMA graft. Acta Cardiol. 2001 Jun; 56(3): 187-8. http://dx.doi.org/10.2143/AC.56.3.2005639 